\title{
Diagnosis and treatment of disseminated intravascular coagulation in COVID-19 patients: a scoping review
}

\author{
Hirotaka Mori $^{1} \cdot$ Hiroshi Ohkawara $^{1} \cdot$ Ryuichi Togawa $^{2} \cdot$ Mami Rikimaru $^{2} \cdot$ Yoko Shibata $^{2} \cdot$ Takayuki Ikezoe $^{1}(\mathbb{C}$
}

Received: 4 September 2020 / Revised: 12 January 2021 / Accepted: 13 January 2021 / Published online: 7 February 2021

(c) Japanese Society of Hematology 2021

\begin{abstract}
Background Disseminated intravascular coagulation (DIC) is noted in severe cases of coronavirus disease 2019 (COVID19). Recently, a number of studies evaluating the diagnosis and treatment of DIC in COVID-19 patients have been reported. Objective The aim of this study is to identify existing gaps where further research is needed on the diagnosis and treatment of DIC complicated by COVID-19.

Methods We used the PRISMA Extension for Scoping Reviews. MEDLINE, CENTRAL, WHO-ICTRP, ClinicalTrial.gov and PROSPERO were searched from their inception to 6 October 2020.

Results Seven studies were selected; five were already published and two are ongoing. DIC was diagnosed using the International Society on Thrombosis and Hemostasis (ISTH) DIC score $(n=4)$ and the sepsis-induced coagulopathy (SIC) DIC score $(n=5)$. Seven studies examined the effectiveness of low molecular weight heparin (LMWH); of these, four studies used a prophylactic dose and five used a therapeutic dose of LMWH. A prophylactic dose of unfractionated heparin (UFH) was investigated in two studies.

Conclusion Studies on DIC diagnostic criteria and anticoagulants were limited to the ISTH or SIC scores and heparinoids, particularly LMWH. Further studies are needed to compare these with other available DIC scoring systems and anticoagulants.
\end{abstract}

Keywords COVID-19 Coagulopathy $\cdot$ Disseminated intravascular coagulation $\cdot$ Anticoagulants $\cdot$ Scoping review

\section{Background}

One of the major clinical manifestations noted in coronavirus disease 2019 (COVID-19) patients is coagulopathy, including disseminated intravascular coagulation (DIC) and thrombosis that is triggered, at least in part, by cytokines produced by inflammatory cells [1]. In addition, the endothelial cell derangement caused by severe acute respiratory syndrome coronavirus 2 (SARS-CoV-2), the pathogen that causes COVID-19, may contribute to the development of coagulopathy; SARS-CoV-2 exploits the angiotensin-converting enzyme 2 (ACE2) receptor expressed on the cell surface of vascular endothelial cells and induces diffuse

Takayuki Ikezoe

ikezoet@fmu.ac.jp

1 Department of Hematology, Fukushima Medical University, 1 Hikarigaoka, Fukushima 960-1295, Japan

2 Department of Respiratory Medicine, Fukushima Medical University, Fukushima 960-1295, Japan endothelial inflammation, so-called endothelialitis [2], resulting in thrombotic microangiopathies [3]. Moreover, SARS-Cov-2 infected neutrophils via ACE2, leading to the release of neutrophil extracellular traps (NETs) which activates the coagulation pathways and platelets [4]. The NETs also cause endothelial cell derangements. This complex process plays a role in the development of the coagulopathy induced by SARS-CoV-2. A previous observational study found that the presence of DIC is associated with poor prognosis in COVID-19 patients [5]. Currently, many papers are focusing on the treatment of COVID-19-associated coagulopathy; however, there is no scoping review on this topic. The scoping review is a useful method to review evidence in rapidly emerging topics. The identification and analysis of knowledge gaps are valuable indications for conducting a scoping review [6]. The objective of this review is to conduct a scoping review to systematically map the DIC diagnosis criteria and anticoagulants used in this area and to identify the existing gaps where further research needs to be performed. 


\section{Methods}

\section{Protocol and registration}

We conducted this scoping review after the a priori protocol was registered in Protocols.io. Link: https://dx.doi. org/10.17504/protocols.io.bgrpjv5n. Please refer to the protocol for detail information of the inclusion and exclusion criteria and the review process. The methodology to conduct scoping reviews by the Joanna Briggs Institute was followed [7], and the results are presented using the Preferred Reporting Items for Systematic Reviews and Meta-Analyses (PRISMA) Extension for Scoping Reviews (PRISMA-ScR) [8].

\section{Results}

The results of the checklist [8] are described in Table 1.

\section{Selection of sources of evidence}

A total of 1552 articles from their inception to 6 October 2020 (MEDLINE, 1037; CENTRAL, 58; ClinicalTrial. gov, 209; WHO-ICTRP, 226; PROSPERO, 22) regarding the diagnosis and treatment of DIC in COVID-19 patients were identified, and 72 studies were selected according to the title and abstract for further full-text review (Fig. 1). Among them, seven articles fulfilled the inclusion criteria were analyzed in the scoping review [9-15]. The results of the literature search strategy are described in Table 2 . There was no previous scoping review on this topic.

\section{Characteristics of the sources of evidence}

The author, status, publication or registration data, location, study design, diagnosis criteria of DIC, anticoagulants, and outcomes of all the included studies are summarized in Fig. 2.

\section{Status, publication or registration data, and location}

Regarding the status of the studies, five studies have been published and two clinical studies are ongoing. The first study was published in March 2020 [9], followed by a study in April $(n=1)$ [10], June $(n=1)$ [11], July $(n=1)$ [12] and September $(n=1)$ [13]. The ongoing studies were registered in May $(n=2)[14,15]$. The studies or protocols were reported from Italy $(n=3)[10,11,15]$, China $(n=2)$ $[9,12]$, USA $(n=1)$ [14] and France $(n=1)[13]$.

\section{Study design}

The study design included case series $(n=4)$ [10-13], retrospective cohort studies $(n=2)[9,12]$, and randomized control trial $(\mathrm{RCT})(\mathrm{n}=2)[14,15]$. None of the studies followed case-control or prospective cohort studies.

\section{Diagnosis criteria of DIC}

DIC was diagnosed using several scales, such as the International Society on Thrombosis and Hemostasis (ISTH) DIC score [16] $(n=4)$ [10-13] and sepsis-induced coagulopathy (SIC) DIC score [17] $(n=5)[9,11,12,14,15]$. The DIC diagnostic criteria released from the Japanese Association for Acute Medicine (JAAM) DIC score [18], Japanese Society on Thrombosis and Hemostasis (JSTH) DIC score [19] or Japanese Ministry of Health and Welfare (JMHW) DIC score [20] were not used.

\section{Anticoagulants}

The anticoagulants therapies used in the studies were a prophylactic dose of unfractionated heparin (UFH) $(n=2)$ $[9,14]$, prophylactic dose of low molecular weight heparin $(\mathrm{LMWH})(n=4)[9,12,13,15]$, therapeutic dose of LMWH $(n=5)$ [10-12, 14, 15], and non-treatment $(n=2)[9,12]$. The therapeutic dose of UFH or rTM was not reported.

\section{Outcomes}

The survival of patients was assessed in six studies [9-12, $14,15]$. The outcomes of clinical practices other than death, such as requiring ICU admission, non-invasive ventilation (NIV), or DIC scores were assessed in four studies [11, 13-15]. The side effects caused by the anticoagulant therapy will be reported in two studies $[14,15]$.

\section{Results of the individual sources of evidence}

Tang et al. [9]

Publication data and location: March 2020 from China. Study design: Retrospective cohort study.

Patients: Ninety-seven out of 449 patients (21.6\%) met the SIC criteria (total score $\geq 4$ ).

Intervention: Enoxaparin 40-60 mg daily or UFH $10,000-15,000$ IU daily for at least 7 days.

Comparisons: No anticoagulants or heparin treatment for less than 7 days. 
Table 1 PRISMA-ScR checklist item, PRISMA-ScR preferred reporting items for systematic reviews and meta-analyses extension for Scoping Reviews

\begin{tabular}{|c|c|c|c|}
\hline Section & Item & PRISMA-ScR checklist item & Reported on page \# or section \\
\hline Title & 1 & Identify the report as a scoping review & Front page \\
\hline \multicolumn{4}{|l|}{ Abstract } \\
\hline Structured summary & 2 & $\begin{array}{l}\text { Provide a structured summary that includes (as } \\
\text { applicable): background, objectives, eligibility } \\
\text { criteria, sources of evidence, charting methods, } \\
\text { results, and conclusions that relate to the review } \\
\text { questions and objectives }\end{array}$ & Abstract \\
\hline \multicolumn{4}{|l|}{ Introduction } \\
\hline Rationale & 3 & $\begin{array}{l}\text { Describe the rationale for the review in the context } \\
\text { of what is already known. Explain why the review } \\
\text { questions/objectives lend themselves to a scoping } \\
\text { review approach }\end{array}$ & Introduction \\
\hline Objectives & 4 & $\begin{array}{l}\text { Provide an explicit statement of the questions and } \\
\text { objectives being addressed with reference to their } \\
\text { key elements (e.g., population or participants, } \\
\text { concepts, and context) or other relevant key ele- } \\
\text { ments used to conceptualize the review questions } \\
\text { and/or objectives }\end{array}$ & Introduction \\
\hline \multicolumn{4}{|l|}{ Methods } \\
\hline Protocol and registration & 5 & $\begin{array}{l}\text { Indicate whether a review protocol exists; state } \\
\text { if and where it can be accessed (e.g., a Web } \\
\text { address); and if available, provide registration } \\
\text { information, including the registration number }\end{array}$ & Method \\
\hline Eligibility criteria & 6 & $\begin{array}{l}\text { Specify characteristics of the sources of evidence } \\
\text { used as eligibility criteria (e.g., years considered, } \\
\text { language, and publication status), and provide a } \\
\text { rationale }\end{array}$ & p. 3 in protocol \\
\hline Information sources & 7 & $\begin{array}{l}\text { Describe all information sources in the search (e.g., } \\
\text { databases with dates of coverage and contact with } \\
\text { authors to identify additional sources), as well as } \\
\text { the date the most recent search was executed }\end{array}$ & pp. $3-4$ in protocol \\
\hline Search & 8 & $\begin{array}{l}\text { Present the full electronic search strategy for at least } \\
1 \text { database, including any limits used, such that it } \\
\text { could be repeated }\end{array}$ & pp. 4-5 in protocol \\
\hline Selection of sources of evidence & 9 & $\begin{array}{l}\text { State the process for selecting sources of evidence } \\
\text { (i.e., screening and eligibility) included in the } \\
\text { scoping review }\end{array}$ & p. 6 in protocol \\
\hline Data charting process & 10 & $\begin{array}{l}\text { Describe the methods of charting data from the } \\
\text { included sources of evidence (e.g., calibrated } \\
\text { forms or forms that have been tested by the team } \\
\text { before their use, and whether data charting was } \\
\text { done independently or in duplicate) and any } \\
\text { processes for obtaining and confirming data from } \\
\text { investigators }\end{array}$ & p. 6 in protocol \\
\hline Data items & 11 & $\begin{array}{l}\text { List and define all variables for which data were } \\
\text { sought and any assumptions and simplifications } \\
\text { made }\end{array}$ & p. 7 in protocol \\
\hline Critical appraisal of individual sources of evidence & 12 & $\begin{array}{l}\text { If done, provide a rationale for conducting a criti- } \\
\text { cal appraisal of included sources of evidence; } \\
\text { describe the methods used and how this informa- } \\
\text { tion was used in any data synthesis (if appropriate) }\end{array}$ & Not applicable \\
\hline Synthesis of results & 13 & $\begin{array}{l}\text { Describe the methods of handling and summarizing } \\
\text { the data that were charted }\end{array}$ & p. 7 in protocol \\
\hline
\end{tabular}


Table 1 (continued)

\begin{tabular}{|c|c|c|c|}
\hline Section & Item & PRISMA-ScR checklist item & Reported on page \# or section \\
\hline \multicolumn{4}{|l|}{ Results } \\
\hline Selection of sources of evidence & 14 & $\begin{array}{l}\text { Give numbers of sources of evidence screened, } \\
\text { assessed for eligibility, and included in the review, } \\
\text { with reasons for exclusions at each stage, ideally } \\
\text { using a flow diagram }\end{array}$ & Table 1 in review \\
\hline Characteristics of sources of evidence & 15 & $\begin{array}{l}\text { For each source of evidence, present characteris- } \\
\text { tics for which data were charted and provide the } \\
\text { citations }\end{array}$ & Fig. 2 in review \\
\hline Critical appraisal within sources of evidence & 16 & $\begin{array}{l}\text { If done, present data on critical appraisal of } \\
\text { included sources of evidence (see item 12) }\end{array}$ & Not applicable \\
\hline Results of individual sources of evidence & 17 & $\begin{array}{l}\text { For each included source of evidence, present the } \\
\text { relevant data that were charted that relate to the } \\
\text { review questions and objectives }\end{array}$ & Result in review \\
\hline Synthesis of results & 18 & $\begin{array}{l}\text { Summarize and/or present the charting results as } \\
\text { they relate to the review questions and objectives }\end{array}$ & Mapping studies in review \\
\hline \multicolumn{4}{|l|}{ Discussion } \\
\hline Summary of evidence & 19 & $\begin{array}{l}\text { Summarize the main results (including an overview } \\
\text { of concepts, themes, and types of evidence avail- } \\
\text { able), link to the review questions and objectives, } \\
\text { and consider the relevance to key groups }\end{array}$ & Discussion in review \\
\hline Limitations & 20 & $\begin{array}{l}\text { Discuss the limitations of the scoping review } \\
\text { process }\end{array}$ & Discussion in review \\
\hline Conclusions & 21 & $\begin{array}{l}\text { Provide a general interpretation of the results with } \\
\text { respect to the review questions and objectives, as } \\
\text { well as potential implications and/or next steps }\end{array}$ & Conclusion in review \\
\hline Funding & 22 & $\begin{array}{l}\text { Describe sources of funding for the included } \\
\text { sources of evidence, as well as sources of funding } \\
\text { for the scoping review. Describe the role of the } \\
\text { funders of the scoping review }\end{array}$ & p. 8 in protocol \\
\hline
\end{tabular}

Outcome: The 28-day mortality of heparin users was lower than that of nonusers in patients with an SIC score $\geq 4$ (40.0 vs. $64.2 \%, p=0.029$ ).

Notes: The number of heparin users and nonusers with SIC scores $\geq 4$ was not reported but 94 patients received LMWH and 5 patients received UFH. Neither therapyrelated side effects nor the study design was reported.

\section{Lodigiani et al. [10]}

Publication data and location: April 2020 from Italy.

Study design: Case series.

Patients: The ISTH DIC score of two patients was 5 or greater.

Interventions: A Patient was treated with nadroparin $5700 \mathrm{IU}$ twice daily and the other patient nadroparin 5700 IU once daily.

Outcome: Two patients were dead.

Note: Eight patients met the ISTH DIC criteria and seven patients died during hospitalization but the dose prescribed to the six patients except the two patients was not described.

\section{Mozzaccaro et al. [11]}

Publication data and location: June 2020 from Italy.

Study design: Case series in ISTH DIC and retrospective cohort in non-overt DIC.

Patients: Two patients were confirmed to have SIC score $\geqq 4$ and ISTH-DIC score $\geqq 5$.

Interventions: Enoxaparin $1 \mathrm{mg} / \mathrm{kg}$ twice daily adjusted for glomerular filtration rate.

Outcome: One patient required NIV continuous positive airway pressure and died for multiorgan failure. The other patient needed NIV and then admission to ICU for mechanical ventilation.

Note: The study design was not reported as a case series. Reviewers judged the study design based on how the results are reported.

Liao et al. [12]

Publication data and location: July 2020 from China.

Study design: Case series and retrospective cohort study. 
Fig. 1 PRISMA flow diagram. PRISMA preferred reporting items for systematic reviews and meta-analyses

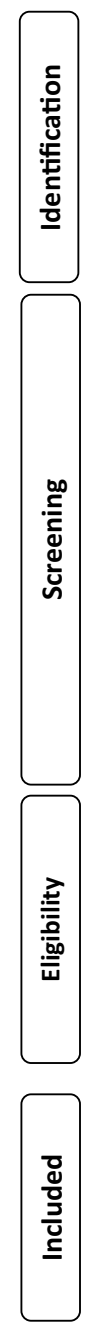

Records identified through database searching Total $(n=1095)$

MEDLINE via PubMed $(\mathrm{n}=1037)$ CENTRAL $(n=58)$
Additional records identified through other sources Total $(n=457)$

$\operatorname{ICTRP}(\mathrm{n}=226)$

ClinicalTrial.gov $(n=209)$ PROSPERO $(n=22)$

\section{Case series}

Patients: Two patients were diagnosed as SIC.

Interventions: LMWH $40 \mathrm{mg}$ once daily.

Outcome: Two patients were discharged.

\section{Cohort study}

Patients: Eight patients were diagnosed as ISTH DIC.

Interventions and comparisons: Five patients were treated with LMWH $40 \mathrm{mg}$ once daily, two LMWH $40 \mathrm{mg}$ twice daily and one without any anticoagulants.

Outcome: All patients died.

Note: The original article reported 8 non-survivors and 12 survivors diagnosed with SIC but the data that could be extracted was limited.
Gerotziafas et al. [13]

Publication data and location: September 2020 from France.

Study design: Case series.

Patients: 49 patients with the ISTH DIC score of 5 points or higher.

Interventions: All patients routinely received thromboprophylaxis with body-weight-adapted enoxaparin.

Outcome: 30 patients required ICU admission.

Note: The dose was not described.

\section{NCT04401293 [14]}

Publication data and location: May 2020 from USA. study design: RCT. 
Table 2 Results of search strategy

No.

\section{MEDLINE}

\#1 "disseminated intravascular coagulation" [MeSH Terms]

\#2 "disseminated intravascular coagulation" [Title/Abstract]

\#3 “disseminated intravascular coagulations" [Title/Abstract]

\#4 "coagulopathy" [Title/Abstract]

\#5 "coagulopathies" [Title/Abstract]

\#6 "Anticoagulants" [MeSH Terms]

\#7 “anticoagulants"[Title/Abstract]

\#8 “anticoagulant" [Title/Abstract]

\#9 “anticoagulation" [Title/Abstract]

\#10 "anticoagulations" [Title/Abstract]

\#11 \#1-\#10/OR

\#12 "Coronavirus" [MeSH Terms]

\#13 “coronavirus" [Title/Abstract]

\#14 "Coronavirus Infections" [MeSH Terms]

\#15 “COVID” [Title/Abstract]

\#16 “cov 2" [Title/Abstract]

\#17 \#7-\#11/OR

\#18 \#10 AND \#17

11,061

10,118

9

13,820

1576

83,353

28,529

47,168

42,726

14

160,193

36,095

34,850

38,656

53,448

18,457

78,193

1037

Cochrane CENTRAL

\#1 MeSH descriptor: [disseminated intravascular coagulation] explode all trees

108

\#2 "disseminated intravascular coagulation":ti,ab

\#3 "disseminated intravascular coagulations":ti,ab

303

\#4 coagulopathy:ti,ab

\#5 coagulopaties:ti,ab

\#6 MeSH descriptor [Anticoagulants] explode all trees

0

1243

\#7 anticoagulants:ti,ab

139

4621

2489

\#9 anticoagulations:ti,ab

4610

\#10 anticoagulation:ti,ab

6

5525

$\# 12 \mathrm{MeSH}$ descriptor [Coronavirus] explode all trees

13,881

\#14 MeSH descriptor [Coronavirus Infections] explode all trees

\#15 COVID:ti,ab

72

611

395

1544

109

1683

58

ClinicalTrial.gov

COVID-19

3662

Other terms: coagulation OR DIC OR coagulopathy OR anticoagulants OR anticoagulation

Total after duplication elimination

209

PROSPERO

\#1 (((coronavirus or corona-virus) AND (wuhan or beijing or shanghai or Italy or South-Korea or korea or China or

Chinese or 2019-nCoV or nCoV or COVID-19 or Covid19 or SARS-CoV* or SARSCov2 or ncov)) OR (pneumonia AND Wuhan) or "COVID-19" or "2019-nCoV" or "SARS-CoV" or SARSCOV2 or 2019-nCov or "2019 coronavirus" or "2019 corona virus" or covid19 or ncov OR "novel corona virus" or "new corona virus" or "nouveau corona virus" or "2019 corona virus" OR "novel coronavirus" or "new coronavirus" or "nouveau coronavirus" or "2019 coronavirus" OR (MeSH DESCRIPTOR Coronavirus Infections EXPLODE ALL TREES) OR ((MeSH

DESCRIPTOR Coronavirus EXPLODE ALL TREES) AND (wuhan or beijing or shanghai or Italy or South-Korea or korea or China or Chinese or 2019-nCoV or nCoV or COVID-19 or Covid19 or SARS-CoV* or SARSCov2 or ncov)))

\#2 disseminated intravascular coagulation" or "coagulation" or "DIC" or "coagulopathy" or "coagulopathy" or

"anticoagulant" 
Table 2 (continued)

No.

WHO-ICTRP

We used the WHO-ICTRP providing COVID-19 trials

Other terms: coagulation OR coagulations OR coagulopathy OR coagulopathy OR coagulants OR anticoagulation

Total

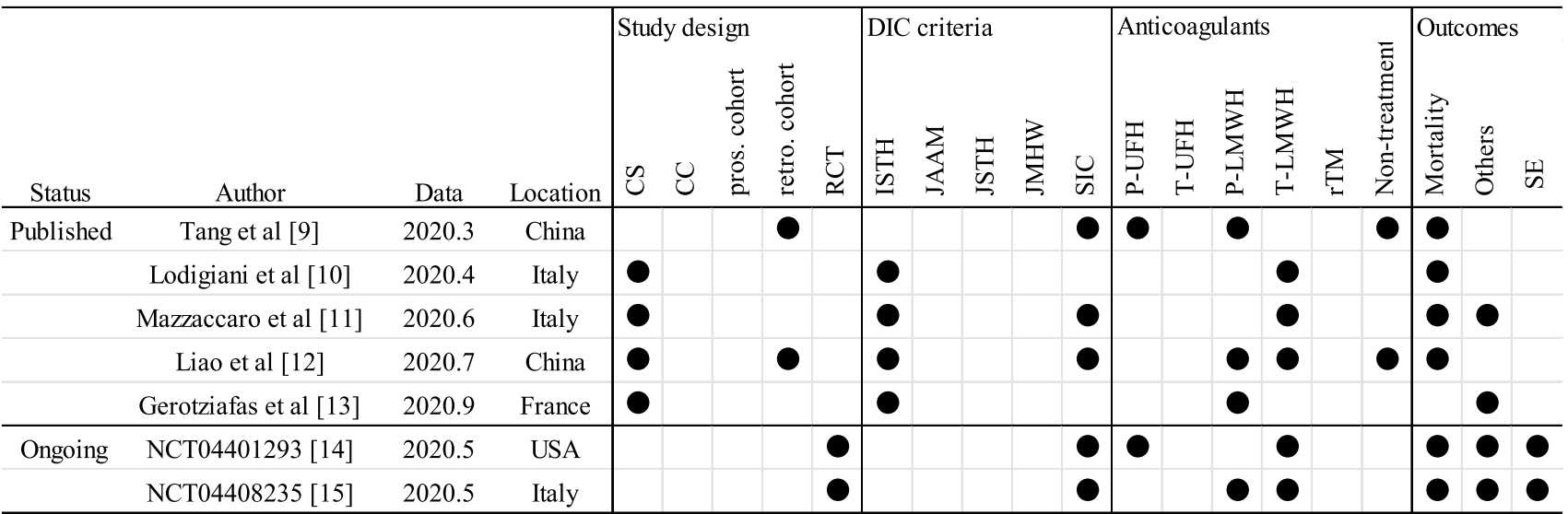

Fig. 2 Characteristics of the sources of evidence. $C R$ case report, $C S$ case series, $C C$ case control, pros prospective cohort study, retro retrospective cohort study, ISTH International Society on Thrombosis and Haemostasis, JAAM Japanese Association for acute medicine, $J M H W$ Japanese Ministry of Health and Welfare, SIC sepsis-induced

Patients: Patients admitted to hospitals with SIC score of $\geq 4$ or D-dimer $>4$ times the upper level of the normal reference range.

Interventions: Therapeutic doses of enoxaparin $1 \mathrm{mg} / \mathrm{kg}$ subcutaneously twice daily adjusted for creatinine clearance during the course of their hospitalization.

Comparison: Prophylactic or intermediate dose of UFH 5000-7500 IU subcutaneously twice or three times daily, enoxaparin 30-40 mg subcutaneously twice or three times daily, dalteparin 2500-5000 IU four times daily.

Outcome: All-cause mortality, SIC score, requiring intubation and major bleeding will be measured.

Note: Whether outcomes for patients with the SIC score $\geq 4$ would be reported was not stated.

\section{NCT04408235 [15]}

Publication data and location: May 2020 from Italy. study design: RCT.

Patients: Inpatients with SIC score $>4$ or D-dimer $>4$ times the upper level of the normal reference range.

Interventions: High dose of enoxaparin $0.7 \mathrm{mg} / \mathrm{kg}$ twice daily. coagulopathy, $P-U F H$ prophylactic dose of unfractionated heparin, $T-U F H$ therapeutic dose of unfractionated heparin, $P-L M W H$ prophylactic dose of low molecular weight heparin, $T-L M W H$ therapeutic dose of low molecular weight heparin, rTM recombinant thrombomodulin, $S E$ side effects, No. number

Comparison: Standard prophylactic dose of enoxaparin $40 \mathrm{mg}$ once a day.

Outcome: All-cause mortality, requiring NIV and major bleeding will be measured.

Note: Whether outcomes for patients with the SIC score $>4$ would be reported was not stated.

\section{Protocol versus overview}

Our planned search strategy registered in protocol.io was compared with the final reported review methods. The search was updated up to October 6, 2020. The inclusion criteria were specifically defined for patients with DIC or SIC diagnosed by scoring systems. There was a difference between the study designs reported by original articles and those judged by our reviews. We reported the study design based on our judgment. 


\section{Discussion}

This study is the first scoping review that maps the available evidence on the diagnosis and treatment of DIC induced by COVID-19 and identified some gaps.

\section{Gaps pertaining to assessment of DIC}

Although multiple scoring systems exist to assess DIC [19], COVID-19 patients treated with anticoagulants have been assessed primarily by the ISTH DIC scores and SIC scores. Unfortunately, none of the studies utilized JAAM, JSTH, and JMHW. Certainly, the accumulation of evidence from a uniform assessment criterion would lead to quantitatively synthesized and reliable conclusions from a meta-analysis. On the other hand, more studies evaluating DIC with more available scoring are needed to elucidate the overall clinical coagulopathy in patients with COID-19.

\section{Gaps pertaining to anticoagulants}

Our results provide insights into the gap that exists among anticoagulants recommended by current therapeutic strategies. Most reviewed studies were related to heparin, especially the prophylactic dose and therapeutic dose of LMWH. A prophylactic dose of UFH or LMWH is not one of the therapeutic strategies to treat DIC caused by severe underlying diseases [21]. However, the prophylactic dose of those anticoagulants was used in five studies. A randomized control trial to compare the prophylactic dose to the therapeutic dose of LMWH is ongoing $[14,15]$.

Another gap pointed out was that no published research evaluating the efficacy of recombinant human soluble thrombomodulin (rTM) was identified. rTM is approved only in Japan for use in daily clinical practice, which could explain why, currently, no study has reported the rTM treatment of COVID-19-induced coagulopathy. However, an international randomized trial to assess the effect of rTM on sepsis-associated coagulopathy was conducted [22]. Compared with $\mathrm{UFH}$, the use of rTM improved the DIC resolution rate in patients with DIC caused by hematological malignancies or infectious diseases [23], and it is the rationale to register a clinical trial comparing rTM with other anticoagulants.

Heparin may possess some anti-inflammatory function as a systematic review showed that the use of heparin decreased the serum levels of inflammatory-related biomarkers in patients with inflammatory diseases [24]. In addition, a high dose of heparin was shown to inhibit the interaction of histone, a major component of NETs, with platelets [25]. Given that the lectin-like domain of rTM possesses antiinflammatory function [26] and rTM degrades histones via activated protein C [27], we assume that the use of rTM is the reasonable option to cope with DIC in CVID-19 patients.

\section{Gaps pertaining to the study design.}

There is also a gap in the study design. Most of the reviewed studies were observational, starting with low-quality evidence, according to the GRADE system for grading the quality of evidence [28], which likely limits the validity and reproducibility of the results. In addition to this gap, three studies did not report the study design, and one reported the design incorrectly and was judged by our reviewers to be a case series because of not comparing the results of anticoagulants. Therefore, future studies that could apply to clinical practice should focus on improving study quality.

\section{Strengths and limitations}

Our review was conducted systematically according to the methodology after registration of a prior protocol. Performance was based on the recommendations from the PRISMA Extension for Scoping Reviews. We believe that this review followed a robust method.

We acknowledge several limitations of our study. Due to the urgent need for evidence on this topic and limited time, we did not contact authors to clarify the details of primary data when necessary. Because studies on this topic are rapidly being conducted, there may be other studies that were not examined in this review when the results are published. Another limitation is that this scoping review only identified future needed studies to clarify the prognosis of patients who received anticoagulants for part of the COVID-19-related coagulopathy. A previous study showed that a significant portion of COVID-19-related coagulopathy was a specific phenotype of coagulopathy which was far from SIC in terms of hemostatic parameters [29]. The authors concluded that the coagulopathy noted in COVID-19 patients might be promoted by the local thrombus [29]. The population covered by this review was limited to patients diagnosed with DIC or SIC and treated with anticoagulants. However, this limitation of the population did not significantly undermine the generalizability of the results in this study. As the previous study also argued that there were no significant differences in the incidence of JAAM DIC and ISTH DIC during the first seven days between the COVID and non-COVID groups. To the best of our knowledge, there was no scoping review that examined the concept of COVID-19-related coagulopathy which is neither DIC nor SIC, and identified the overall COVID-19-related coagulopathy including the specific phenotype of coagulopathy and typical DIC and SIC. Therefore, to identify gaps in comparison with existing evidence on anticoagulants [30], it was necessary and appropriate to limit the population of this review to DIC 
patients for whom definitions have been established. Future scoping reviews should be conducted to examine the definition of the overall COVID-19-related coagulopathy and to investigate the prognosis of anticoagulants for COVID19-related coagulopathy.

\section{Conclusion}

Evidence on the treatment of DIC in COVID-19 patients is accumulating but is still mainly focused on the assessment of DIC by the score of ISTH DIC or SIC, the use of heparinoids, especially LMWH and observational study designs. The evidence found on this topic was not qualitatively or quantitatively sufficient to conduct a systematic review that would provide practical guidance. Further clinical trials are needed to compare other available DIC scoring systems and anticoagulants and may provide more accurate results by improving their study design.

Recently, ISTH has released the interim guidance on recognition and management of coagulopathy in COVID-19; it recommends the physicians to measure D-dimer, PT, and the platelet count to determine the patients requiring admission. Based on the reports by Tang et al. [8], the prophylactic dose of LMWH is recommended for all admitted patients [29]. This guidance should be modified after the accumulation of more solid evidences.

Acknowledgements This study was supported by KAKENHI (18H02844) to T. Ikezoe.

Author contributions HM conceived this study and designed the protocol. HM and $\mathrm{HO}$ developed and conducted the literature search strategy. HM, HO, RT, MR, YS, and TI drafted and revised the manuscript.

\section{Compliance with ethical standards}

Conflict of interest All of the authors declare that they have no conflicts of interests.

\section{References}

1. Walborn A, Hoppensteadt D, Syed D, Mosier M, Fareed J. Biomarker profile of sepsis-associated coagulopathy using biochip assay for inflammatory cytokines. Clin Appl Thromb Hemost. 2018;24:625-32.

2. Varga Z, Flammer AJ, Steiger P, Haberecker M, Andermatt R, Zinkernagel AS, et al. Endothelial cell infection and endotheliitis in COVID-19. Lancet. 2020;395:1417-8.

3. Gavriilaki E, Brodsky RA. Severe COVID-19 infection and thrombotic microangiopathy: success does not come easily. Br J Haematol. 2020;189:e227-30.

4. Veras FP, Pontelli MC, Silva CM, Toller-Kawahisa JE, de Lima M, Nascimento DC, et al. SARS-CoV-2-triggered neutrophil extracellular traps mediate COVID-19 pathology. J Exp Med. 2020;217:e20201129.

5. Tang N, Li D, Wang X, Sun Z. Abnormal coagulation parameters are associated with poor prognosis in patients with novel coronavirus pneumonia. J Thromb Haemost. 2020;18:844-7.

6. Munn Z, Peters MDJ, Stern C, Tufanaru C, McArthur A, Aromataris E. Systematic review or scoping review? Guidance for authors when choosing between a systematic or scoping review approach. BMC Med Res Methodol. 2018;18:143.

7. Peters MDJ GC, Mcinerney P, Soares CB, Khalil H, Parker D. The Joanna Briggs Institute reviewers' manual 2015: Methodology for JBI scoping reviews. https://nursing.lsuhsc.edu/JBI/docs/ ReviewersManuals/Scoping-.pdf

8. Tricco AC, Lillie E, Zarin W, O'Brien KK, Colquhoun H, Levac $\mathrm{D}$, et al. PRISMA extension for scoping reviews (PRISMA-ScR): checklist and explanation. Ann Intern Med. 2018;169:467-73.

9. Tang N, Bai H, Chen X, Gong J, Li D, Sun Z. Anticoagulant treatment is associated with decreased mortality in severe coronavirus disease 2019 patients with coagulopathy. J Thromb Haemost. 2020;18:1094-9.

10. Lodigiani C, Iapichino G, Carenzo L, Cecconi M, Ferrazzi P, Sebastian T, et al. Venous and arterial thromboembolic complications in COVID-19 patients admitted to an academic hospital in Milan. Italy Thromb Res. 2020;191:9-14.

11. Mazzaccaro D, Giacomazzi F, Giannetta M, Varriale A, Scaramuzzo R, Modafferi A, et al. Non-overt coagulopathy in nonICU Patients with mild to moderate COVID-19 pneumonia. J Clin Med. 2020;9:1781.

12. Liao D, Zhou F, Luo L, Xu M, Wang H, Xia J, et al. Haematological characteristics and risk factors in the classification and prognosis evaluation of COVID-19: a retrospective cohort study. Lancet Haematol. 2020;7:e671-8.

13. Gerotziafas GT, Sergentanis TN, Voiriot G, Lassel L, Papageorgiou C, Elabbadi A, et al. Derivation and validation of a predictive score for disease worsening in patients with COVID-19. Thromb Haemost. 2020;120:1680-90.

14. NCT04401293. Full dose heparin vs. prophylactic or intermediate dose heparin in high risk COVID-19 patients. http://clinicaltrials. gov/show/NCT04401293. 2020.

15. NCT04408235. Randomised controlled trial comparing efficacy and safety of high versus low low-molecular weight heparin dosages in hospitalized patients with severe COVID-19 pneumonia and coagulopathy not requiring invasive mechanical ventilation (COVID-19 HD): a structured summary of a study protocol. 2020;21:574. http://clinicaltrials.gov/ct2/show/NCT04408235.

16. Taylor FB Jr, Toh CH, Hoots WK, Wada H, Levi M. Towards definition, clinical and laboratory criteria, and a scoring system for disseminated intravascular coagulation. Thromb Haemost. 2001;86:1327-30.

17. Iba T, Nisio MD, Levy JH, Kitamura N, Thachil J. New criteria for sepsis-induced coagulopathy (SIC) following the revised sepsis definition: a retrospective analysis of a nationwide survey. BMJ Open. 2017;7:e17046.

18. Gando S, Wada H, Asakura H, Iba T, Eguchi Y, Okamoto K, et al. Evaluation of new Japanese diagnostic criteria for disseminated intravascular coagulation in critically ill patients. Clin Appl Thromb Hemost. 2005;11:71-6.

19. Asakura H, Takahashi H, Uchiyama T, Eguchi Y, Okamoto $\mathrm{K}$, Kawasugi K, et al. Proposal for new diagnostic criteria for DIC from the Japanese Society on Thrombosis and Hemostasis. Thromb J. 2016;14:42.

20. Kobayashi N, Maekawa T, Takada M, Tanaka H, Gonmori H. Criteria for diagnosis of DIC based on the analysis of clinical and laboratory findings in 345 DIC patients collected by the Research Committee on DIC in Japan. Bibl Haematol. 1983:265-75. 
21. Levi M, Toh CH, Thachil J, Watson HG. Guidelines for the diagnosis and management of disseminated intravascular coagulation British Committee for Standards in Haematology. Br J Haematol. 2009; 145:24-33.

22. Vincent JL, Francois B, Zabolotskikh I, Daga MK, Lascarrou JB, Kirov MY, et al. Effect of a recombinant human soluble thrombomodulin on mortality in patients with sepsis-associated coagulopathy: the SCARLET randomized clinical trial. JAMA. 2019;321:1993-2002.

23. Saito H, Maruyama I, Shimazaki S, Yamamoto Y, Aikawa N, Ohno R, et al. Efficacy and safety of recombinant human soluble thrombomodulin (ART-123) in disseminated intravascular coagulation: results of a phase III, randomized, double-blind clinical trial. J Thromb Haemost. 2007;5:31-41.

24. Mousavi S, Moradi M, Khorshidahmad T, Motamedi M. Antiinflammatory effects of heparin and its derivatives: a systematic review. Adv Pharmacol Sci. 2015;2015:507151.

25. Fuchs TA, Bhandari AA, Wagner DD. Histones induce rapid and profound thrombocytopenia in mice. Blood. 2011;118:3708-14.

26. Ikezoe T. Advances in the diagnosis and treatment of disseminated intravascular coagulation in haematological malignancies. Int $\mathbf{J}$ Hematol. 2021;113:34-44.
27. Xu J, Zhang X, Pelayo R, Monestier M, Ammollo CT, Semeraro F, et al. Extracellular histones are major mediators of death in sepsis. Nat Med. 2009;15:1318-21.

28. Guyatt GH, Oxman AD, Vist GE, Kunz R, Falck-Ytter Y, Alonso-Coello P, et al. GRADE: an emerging consensus on rating quality of evidence and strength of recommendations. BMJ. 2008;336:924-6.

29. Umemura Y, Yamakawa K, Kiguchi T, Nishida T, Kawada M, Fujimi S. Hematological phenotype of COVID-19-induced coagulopathy: far from typical sepsis-induced coagulopathy. J Clin Med. 2020;9:2875.

30. Murao S, Yamakawa K. A systematic summary of systematic reviews on anticoagulant therapy in sepsis. J Clin Med. 2019;8:1869.

Publisher's Note Springer Nature remains neutral with regard to jurisdictional claims in published maps and institutional affiliations. 